Design Cross-sectional study.

Participants An online questionnaire based on the 5th Consensus Statement on Concussion in Sport (Berlin, 2016) and The FA concussion guidelines was distributed by The FA and the Professional Game Match Officials Limited (PGMOL) to their referee members (Queen Mary University of London Research Etics Committee QMREC2014/24/162)

Main Results 208 questionnaires were completed of which 34 $(16 \%)$ were from referees officiating in top two tiers of English football (Premier League and Championship). 48\% felt confident recognising SRC on pitch, $62 \%$ confident in the immediate management of concussion, and 69\% confident that their concussion knowledge was adequate to officiate games. Some concussion-related symptoms had high awareness, but seizure and aggression had lower recognition. 32\% incorrectly believed only a direct blow to the head could cause a concussion. $30 \%$ felt the final say on player removal was not that of the medical team. Low rates of concussion specific education or training were found, but high interest in future education.

Conclusions Improvement in concussion knowledge amongst football referees is required. An emphasis on educational initiatives aimed at these stakeholders could improve the health and welfare of those participating in football.

\section{PLAYING THE GAME OR GAMING THE SYSTEM: ARE US PRIVATE HIGH SCHOOL STUDENT-ATHLETES REPORTING, HIDING OR FAKING CONCUSSIONS?}

${ }^{1}$ Katherine Snedaker, ${ }^{2}$ Jason Bouton. ${ }^{1}$ PINK Concussions, Norwalk, CT, USA; ${ }^{2}$ KING School, Stamford, CT, USA

\subsection{6/bjsports-2021-IOC.298}

Background Sports- and recreation-related (SRR) activities are a major cause of concussions among adolescents. Most adolescent SRR concussion research has been conducted among public school students. As private schools are qualitatively different from public schools (e.g. location, socioeconomic status, sports played), this study explores the concussion experiences of a large group of private high school students.

Objective The purpose of this study is to describe concussion reporting and return to learn (RTL) and return to play (RTP) post-concussion in a sample of private high school students who play a sport or engage in a recreational activity. These outcomes were also stratified by gender, contact level of their primary sport, and grade.

Design We surveyed students who played sports or a recreational activity about the sports they played, and their selfreported concussion experiences between April - May, 2018. Descriptive, bivariate, and multivariate statistics are presented. Setting Ten New England private preparatory high schools. Patients (or Participants) Data was collected from 2,122 male and female students.

Interventions (or Assessment of Risk Factors) Sex, grade, contact level of primary sport played and age of first concussion. Main Outcome Measurements The main outcome of interest was percentage of students who reported/hid a concussion, and the time it the student to return to school and then to sport.

Results One-third (33.0\%) of students who reported engaging in sport-activities, self-reported experiencing a concussion in their lifetime. A higher percentage of males, students who played contact sports, and those who played multiple seasons of school sports, reported a concussion. Sex, contact level of primary sport played and age of first concussion were also significantly associated with reporting a sports-related concussion. 3\% of students reported faking concussions.

Conclusions A sizeable proportion of private preparatory high school students reported experiencing a concussion, with some students at higher risk.

\section{LOWER DYNAMIC NECK STRENGTH IS ASSOCIATED WITH HISTORY OF CONCUSSION IN VARSITY FEMALE SOCCER PLAYERS}

Theo Versteegh. Western University, London, Canada

\subsection{6/bjsports-2021-IOC.299}

Background There is mounting evidence that dynamic neck strength may play a role in protecting against concussion. It is also well established that athletes with a prior history of concussion are at higher risk than those with no prior history.

Objective To assess if there is a difference in dynamic neck strength between athletes with a self-declared history of concussion $(\mathrm{HxC})$ and athletes with no history of concussion (No-HxC). Secondly, to determine if dynamic neck strength can be used as a predictor for previous concussion history through a receiver operating characteristic curve (ROC) and hence, be used as a proxy for future concussion risk.

Design Observational cohort design

Setting Varsity level female competitive soccer players

Participants 28 athletes (average age 19.4 years, range 18-21), separated by self-declared history of concussion ( $\mathrm{HxC} n=10$ and No-HxC $\mathrm{n}=18$ )

Assessment Dynamic neck strength was calculated as the peak Rate of Force Development (RFD) in pounds-force per second $\left(\mathrm{lb}_{\mathrm{f}}{ }^{*} \mathrm{~s}^{-1}\right)$ achieved during 50 revolutions on the TopSpin360 neuromuscular neck-training device.

Results RFD for $\mathrm{HxC}$ was $3.85 \mathrm{lb}_{\mathrm{f}}{ }^{*} \mathrm{~s}^{-1}$ (95\% CI $2.53-5.17$ $\mathrm{lb}_{\mathrm{f}}{ }^{*} \mathrm{~s}^{-1}$ ) while RFD for No-HxC was $7.14 \mathrm{lb}_{\mathrm{f}}{ }^{*} \mathrm{~s}^{-1}$ (95\% CI $5.17-9.12 \mathrm{lb}_{\mathrm{f}}{ }^{*} \mathrm{~s}^{-1}$ ) Independent samples t test $\mathrm{p}=0.012$. ROC cut-off value of $4.5 \mathrm{lb}_{\mathrm{f}}{ }^{*} \mathrm{~s}^{-1}$ provides a sensitivity of $72 \%$ and specificity of $80 \%$ for detecting those with a history of concussion.

Conclusions In this pilot study of varsity female soccer athletes, those with a history of concussion demonstrate significantly lower dynamic neck strength measurements compared to teammates with no history of concussion. Knowing that $\mathrm{HxC}$ athletes are at higher risk of future concussion, the ROC cut-off value of $4.5 \mathrm{lb}_{\mathrm{f}}{ }^{*} \mathrm{~s}^{-1}$ provides a starting point for future studies using dynamic neck strength values for assessing baseline concussion risk in athletes.

\section{THE ROLE OF NECK STRENGTH IN MITIGATING SPORT RELATED CONCUSSION: A SYSTEMATIC REVIEW AND META-ANALYSIS}

${ }^{1}$ Theo Versteegh, ${ }^{2}$ Elliott Jonny, ${ }^{7}$ lan Gilchrist, ${ }^{3,8}$ Neil Heron, ${ }^{4}$ Pooler Archbold, ${ }^{5}$ Michael Webb, ${ }^{6}$ Kerry Peek. 'Western University, London, Canada; ${ }^{2}$ Ulster Hospital, Belfast, Ireland; ${ }^{3}$ Centre for Public Health, Belfast, Ireland; ${ }^{4}$ Royal Victoria Hospital, Belfast, Ireland; ${ }^{5}$ Ulster Rugby, Belfast, Ireland; ${ }^{6}$ University of Sydney, Sydney, Australia; ${ }^{7}$ Queen's University, Kingston, Canada; ${ }^{8}$ Keele University, Staffordshire, UK

10.1136/bjsports-2021-IOC.300 